\title{
NAVILAS Laser System Focal Laser Treatment for Diabetic Macular Edema - One Year Results of a Case Series
}

Jesse J. Jung ${ }^{*}, 1,2,3,4$, Roberto Gallego-Pinazo ${ }^{1,2,5}$, Antonio Lleó-Pérez ${ }^{6}$, Jonathan I. Huz ${ }^{1}$ and Irene A. Barbazetto $1,2,3,4$

\author{
${ }^{I}$ Department of Ophthalmology, New York University School of Medicine, New York, NY, USA \\ ${ }^{2}$ Vitreous Retina Macular Consultants of New York, New York, NY, USA \\ ${ }^{3}$ LuEsther T. Mertz Retinal Research Center, Manhattan Eye, Ear, and Throat Hospital, New York, NY, USA \\ ${ }^{4}$ Edward S. Harkness Eye Institute, Columbia University College of Physicians and Surgeons, New York, NY, USA \\ ${ }^{5}$ Department of Ophthalmology, University and Polytechnic Hospital La Fe, Valencia, Spain \\ ${ }^{6}$ Department of Ophthalmology, Hospital Francesc de Borja, Gandía, Spain
}

\begin{abstract}
Purpose: To report one year outcomes of focal Navigated Retina Laser Therapy (NAVILAS) for diabetic macular edema (DME).

Methods: Retrospective cohort series of 7 diabetic patients treated with NAVILAS focal laser. Statistical analysis included descriptive and continuous variables (Best-corrected logMAR Visual Acuity and time-domain optical coherence tomography (OCT) parameters) which were compared using a non-parametric procedure, the Friedman tests for repeated measures. A p-value of less than 0.05 was considered to denote statistical significance.

Results: 7 diabetic patients (4 male; 3 female) with an average age of 60.8 years (range $48-85$ years) were included. All treated eyes were phakic; patients had an average hemoglobin A1C of 9.1 (range 7.8-11.7) at baseline and 8.0 (range 7.48.4) at 12 months. Six of the 7 patients had intravitreal bevacizumab injections prior to focal laser treatment with 1 patient having had more than 1 prior injection (total 3). At 12 months, median logMAR improved from 0.695 ( \pm interquartile range 0.574$)$ to 0.477 ( $\pm 0.573, p<0.001)$. OCT median central foveal thickness decreased from $248( \pm 112)$ to $220 \mu \mathrm{m}( \pm$ $41, p<0.001)$; total macular volume decreased from $7.84( \pm 0.8)$ to $7.44 \mathrm{~mm}^{3}( \pm 0.7, p=0.117)$; and largest macular subfield thickness decreased from $354( \pm 116)$ to $289 \mu \mathrm{m}( \pm 42, p<0.001)$. All patients were treated without complications.

Conclusions: Focal NAVILAS showed to be safe and effective in treating DME with improvement in visual acuity and macular edema on OCT over 12 months in this case series. In clinical practice, combined treatment with focal laser including NAVILAS and anti-vascular endothelial growth factor may provide long-term improvement in DME.
\end{abstract}

Keywords: Anti-VEGF, bevacizumab, diabetes, focal laser photocoagulation, macular edema, NAVILAS.

\section{INTRODUCTION}

Laser photocoagulation has been the standard of care for patients with clinically significant macular edema for several decades, but with several new advancements in treatment modalities including anti-vascular endothelial growth factor (VEGF), our ability to treat diabetic macular edema (DME) has significantly improved. The Early Treatment Diabetic Retinopathy Study (ETDRS) first showed the efficacy of focal laser therapy for treatment of DME [1], and now with the introduction of intravitreal anti-VEGF therapy with ranibizumab (Lucentis, Genentech Inc., San Francisco, CA, USA) and bevacizumab (Avastin, Genentech Inc., San

*Address correspondence to this author at the Department of Ophthalmology 462 First Avenue, NBV 5N 18 (Bellevue Hospital Building), New York, NY 10016, USA; Tel: 212-263-6434;

Fax: 212-263-8749; E-mail: jung.jesse@gmail.com
Francisco, CA, USA) as seen in the ranibizumab for diabetic macular edema (RISE) and ranibizumab injection in subjects with clinically significant macular edema with center involvement secondary to diabetes mellitus (RIDE), prevention of further vision loss from macular edema has been substantial [2,3]. Although intravitreal anti-VEGF therapy has become one of the mainstays in treatment of DME, focal laser therapy remains important in treating extrafoveal DME, and individualized treatments with both modalities in clinical practice may become the future of DME care.

The ETDRS study as well as the Diabetic Retinopathy Clinical Research (DRCR) network have provided clinicians with guidelines for the treatment of DME, but there is little standardization or quality control when it comes to clinical practice. In addition, accuracy of the treatment is always a concern especially when applying focal laser treatment near the fovea. The navigated laser photocoagulator (NAVILAS; 
OD-OS GmbH, Teltow, Germany) is a new prototype of retinal eye-tracking laser delivery system with integrated digital fundus imaging (live color fundus photographs, redfree and infrared imaging, and fluorescein angiography) which could potentially increase the effectiveness and efficiency of focal laser therapy for DME by aligning preplanned treatment maps with real-time fundus images. This scanning slit-lamp based system allows registered image overlay and laser stabilization on the retina using image processing in the treatment of retinal lesions [4-7]. The system uses a diode-pumped solid-state laser $(532 \mathrm{~nm})$ for both single-spot and pattern retinal photocoagulation [4]. Eye-tracking mechanisms of the instrument compensate for the eye's excursions during treatment and utilize registration of retinal vessel positions and multiple retinal landmarks to limit the possibility of inadvertent placement of laser burns [4-7]. A contact lens can be used but is not necessary for accurate treatment. The physician can use either infrared or full color illumination to follow and modify the positioning of each marked laser spot as the system progresses during its treatment $[6,7]$.

The aim of this study was to investigate the long-term results (12 months) of NAVILAS focal photocoagulation for a group of patients with poorly controlled diabetic macular edema who were also treated with anti-VEGF therapy.

\section{METHODS}

New York University School of Medicine Institutional Review Board (IRB) approval was obtained for this Health Insurance Portability and Accountability Act-compliant, retrospective cohort study and all research adhered to the tenets of the Declaration of Helsinki (IRB \#S12-00331). The medical records of 7 diabetic patients with clinically significant, diabetic macular edema treated with a navigated laser photocoagulator at the Bellevue Eye Clinic were reviewed. Patients were selected for focal laser treatment based on the presence of macular edema on OCT imaging and/or leakage on fluorescein angiography. Each treatment plan was individualized based on the number and size of microaneurysms seen on color fundus photography and fluorescein angiography. The treatments were performed without contact lens and without topical anesthesia. The laser parameters used in this study were a spot size of 100$150 \mu \mathrm{m}$, duration of 100-150 milliseconds (ms), and power between 50-180 milliwatts ( $\mathrm{mW}$ ).

Best-corrected $\log$ MAR (BCVA) and time-domain optical coherence tomography (OCT) (Stratus OCT, Carl Zeiss-Meditec Inc, Dublin, CA) parameters such as central foveal thickness (CFT), total macular volume (TMV), and largest subfield of macular thickness (LMT) were recorded at 3 month intervals over a 12 month follow-up.

Statistical analyses were carried out by one physician (A. L-P) and performed utilizing SPSS 21.0 (IBM Corp). The descriptive statistics are presented as median and interquartile range (IQR) values where available and measured ranges indicate minimums and maximums. Continuous variables for the entire cohort (BCVA and timedomain OCT parameters) were compared using a nonparametric procedure, the Friedman tests for repeated measures, after verifying that our sample was not normally distributed (by applying the Kolmogorov-Smirnov test) neither was there homogeneity in group variances (using the Levene's robust test). A p-value of less than 0.05 was considered to denote statistical significance.

\section{RESULTS}

We treated 7 diabetic patients (4 male; 3 female) with an average age of 60.8 years ( \pm standard deviation 12.3, range 48-85 years). All eyes were phakic; patients had an average hemoglobin A1c of 9.1 ( \pm 1.8 , range 7.8-11.7) at baseline and $8.0( \pm 0.5$, range 7.4-8.4) at 12 months, and these differences were not significant $(p=0.180)$ using the Wilcoxon Signed Ranks Test. Three patients were treated for hypertension and 1 patient showed history of hyperlipidemia. Average systolic blood pressure was $130.1( \pm 16.7$, range $115-159)$ and average diastolic blood pressure was 79.4 ( \pm 15 , range 59-108). Six of the 7 eyes had intravitreal bevacizumab injections prior to focal laser treatment with 1 eye having had more than 1 prior injection (total 3). Two patients had a single treatment of manual focal laser prior to NAVILAS focal laser (Table 1). After 12 months of followup, 3 eyes required further intravitreal bevacizumab injections after NAVILAS focal laser treatment with each eye requiring 6,10 , and 3 injections at intervals of 34 days, 21 days, and 263 days after focal treatment, respectively. Average interval of required bevacizumab injection after NAVILAS treatment was 106 days ( \pm 136.1 , range 21-263).

Table 1. Baseline Demographic and Clinical Characteristics of Patients Studied

\begin{tabular}{|c|c|}
\hline & Demographic Characteristics \\
\hline Number of Patients & 7 \\
\hline Age (mean) & 60.8 \\
\hline Age (range) & $48-85$ \\
\hline$\#$ of OD & 4 \\
\hline$\#$ of OS & 3 \\
\hline HTN & 3 \\
\hline HLD & 1 \\
\hline Hgb A1c (mean) & 9.1 \\
\hline Hgb A1c (range) & $7.8-11.7$ \\
\hline Systolic BP (mean) & 130.1 \\
\hline Systolic BP (range) & $115-159$ \\
\hline Diastolic BP (mean) & 79.4 \\
\hline Diastolic BP (range) & $59-108$ \\
\hline Prior Focal & 2 \\
\hline Prior Bevacizumab & 6 \\
\hline
\end{tabular}

All laser applications hit the pre-planned microaneurysm points of focal leakage in all eyes (Fig. 1E, F). Color fundus photos and fluorescein angiography was performed before (Fig. 1A, C) and after (Fig. 1B, D) treatment and showed 


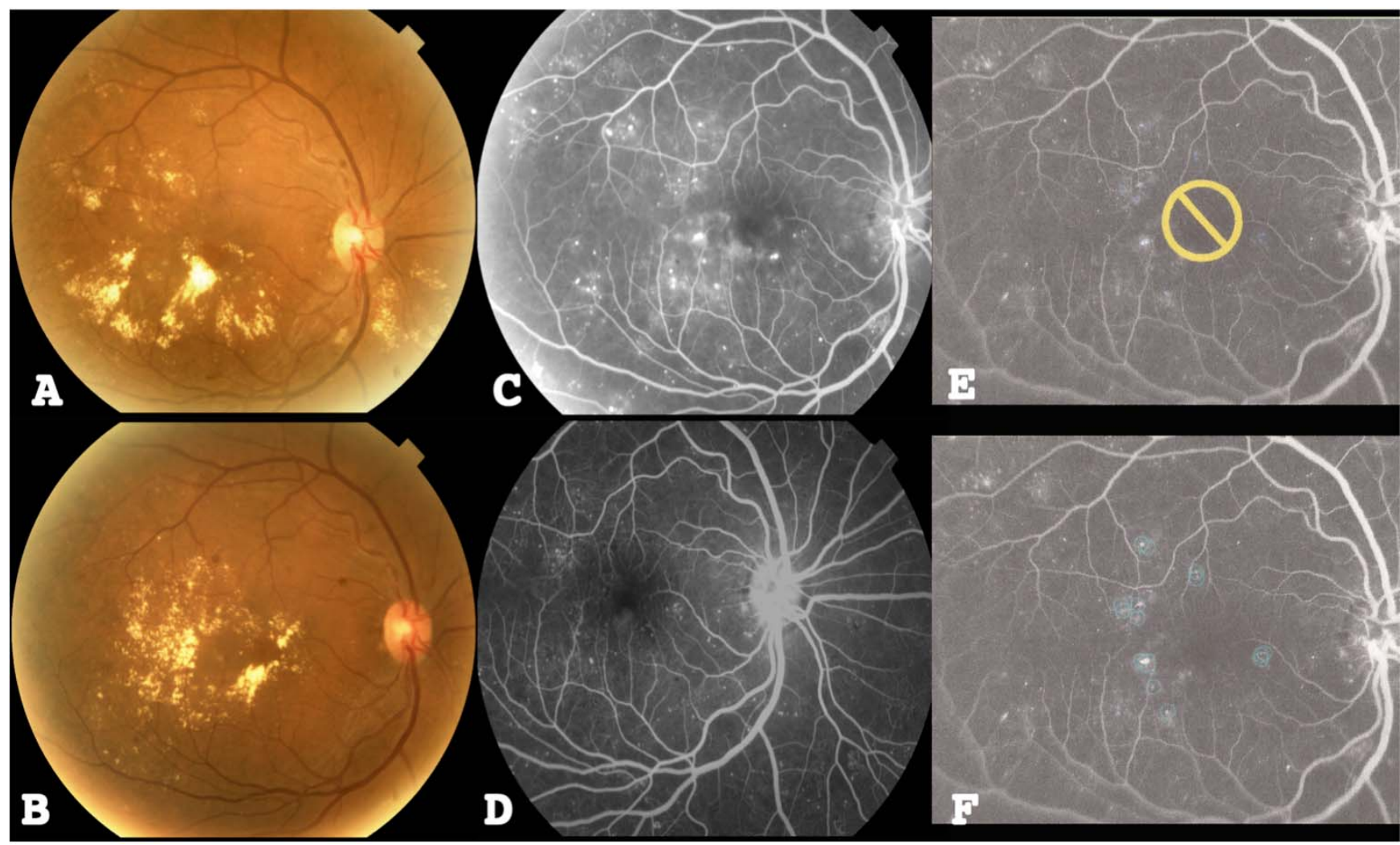

Fig. (1). (A) Color fundus photo of the right eye of a study patient with DME before treatment. (B) Color fundus photo of the left eye after treatment. (C) Represents early fluorescein angiogram (FA) of the right eye prior to NAVILAS treatment. (D) Represents early (FA) of the right eye after treatment. (E, F) An image overlay composed and displayed over the pre-treatment FA showing planning elements of microaneurysm treatments and no-treatment zones.

improvement in clinical features such as amount of hard exudate, number of leaking microaneurysms, and nonproliferative diabetic retinopathy. Average number of shots was 35.8 ( \pm standard deviation 19.7, range 12-70); average amount of energy was $94.8 \mathrm{~mW}( \pm 26.2$, range $50-146)$; average pulse duration was $99.2 \mathrm{~ms}$ ( \pm 3.6, range 90-103); average spot size was $91.3 \mu \mathrm{m}$ ( \pm 29.8 , range $50-140)$; and average total amount of energy administered to the eye during treatment was 2711.5 Joules $( \pm 1896.2$, range $714-$ 5580). There were no complications or inadvertent photocoagulation of the fovea or optic nerve head.

Before treatment, BCVA of all eyes showed a median $( \pm$ IQR) of $0.695 \pm 0.5$, and OCT parameters were $248 \pm 112$ $\mu \mathrm{m}$ for CFT, $7.84 \pm 0.8 \mathrm{~mm}^{3}$ for TMV and $354 \pm 116 \mu \mathrm{m}$ for LMT. At 12 months, comparing these variables to baseline, all participants' BCVA improved to $0.477 \pm 0.5$ and OCT parameters: CFT decreased to $220 \pm 41 \mu \mathrm{m}$; TMV decreased to $7.44 \pm 0.7 \mathrm{~mm}^{3}$ and LMT decreased to $289 \pm 42 \mu \mathrm{m}$, as can be observed in Table $\boldsymbol{2}$. These differences were statistically significant for BC LogMAR VA $(p<0.001)$, OCT Central Foveal Thickness $(p<0.001)$ and OCT Largest Deviation $(p<0.001)$ and the ranks of these variables differ significantly from their expected value in the repeated measures with the Friedman chi-square tests, as can be observed in Table 3 .

An example of one patient's OCT macular topographic map before (Fig. 2A, B) and macular cross-section OCT (Fig. 2C) and macular topographic map after (Fig. 2D, E) and macular cross-section OCT (Fig. 2F) showed a decrease in DME.

Table 2. Analysis of Optical Coherence Tomography Parameters and Best-corrected LogMAR Visual Acuity

\begin{tabular}{|c|c|c|c|c|}
\hline & BCVA LogMAR & OCT Central Foveal Thickness & OCT Macular Volume & OCT Largest Deviation \\
\hline \hline Baseline & $0.695 \pm 0.574$ & $248 \pm 112$ & $7.84 \pm 0.874$ & $354 \pm 116$ \\
\hline 3 months follow-up & $0.544 \pm 0.447$ & $258 \pm 100$ & $7.92 \pm 0.630$ & $354 \pm 76$ \\
\hline 6 months follow-up & $0.397 \pm 0.505$ & $288 \pm 163$ & $7.88 \pm 0.559$ & $346 \pm 93$ \\
\hline 9 months follow-up & $0.477 \pm 0.367$ & $288 \pm 130$ & $7.60 \pm 1.315$ & $312 \pm 116$ \\
\hline 12 months follow-up & $0.477 \pm 0.573$ & $220 \pm 41$ & $7.44 \pm 0.757$ & $289 \pm 42$ \\
\hline
\end{tabular}

Statistical median \pm interquartile range of best corrected LogMAR visual acuity (BCVA LogMAR) and optical coherence tomography parameters (OCT) from baseline to each 3 month interval over 12 months in the entire sample. 
Table 3. Statistical Analysis of Means by Friedman Chi-Square Test

\begin{tabular}{|c|c|c|c|c|}
\hline & BCVA LogMAR & OCT Central Foveal Thickness & OCT Total Macular Volume & OCT Largest Deviation \\
\hline \hline Chi-Square value & 10.939 & 33.000 & 2.455 & 33.000 \\
\hline Asymptotic significance & $<0.001$ & $<0.001$ & 0.117 & $<0.001$ \\
\hline
\end{tabular}

Friedman repeated measures chi-square tests of best corrected LogMAR visual acuity (BCVA) and optical coherence tomography parameters (OCT) for the entire sample.

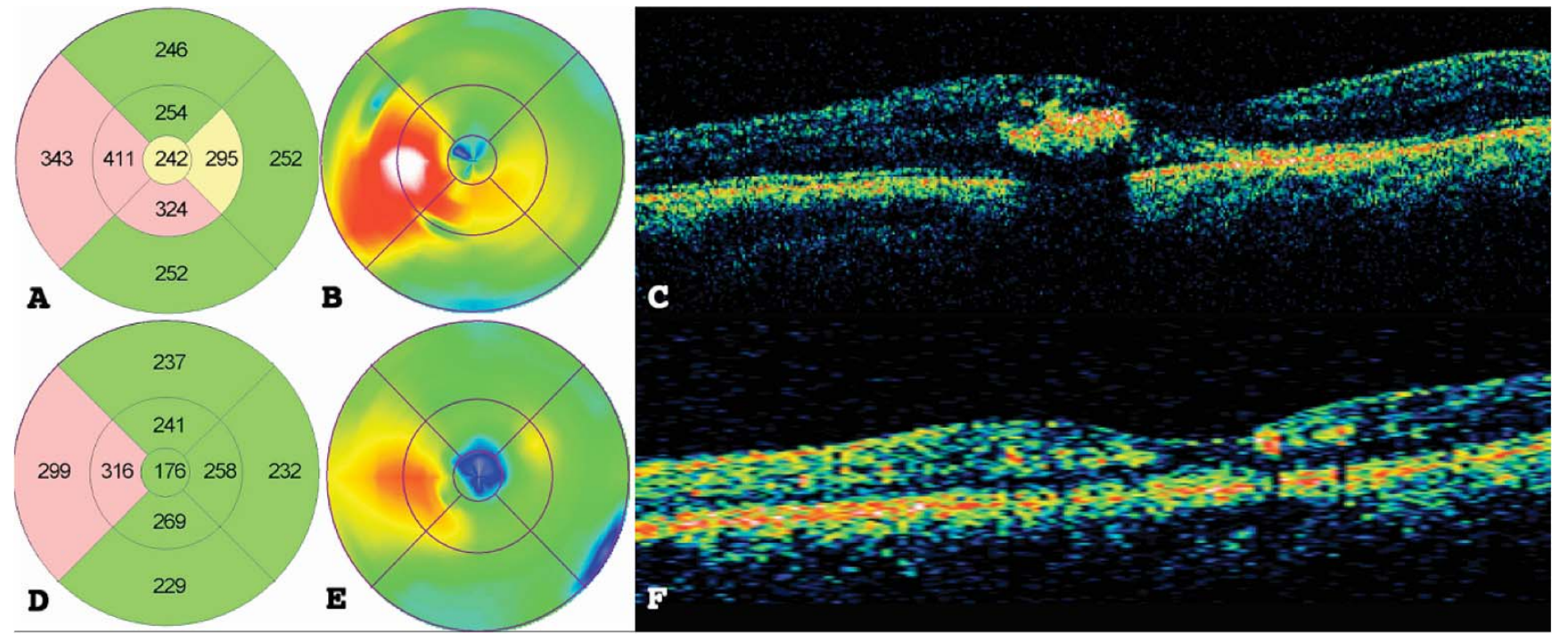

Fig. (2). (A) Macular topographic map, (B) color macular map, and (C) macular cross-section optical coherence tomography (OCT) of the same study patient from Fig. (1) before treatment. (D) Macular topographic map, (E) color macular map, and (F) macular cross-section OCT after NAVILAS treatment.

\section{DISCUSSION}

Focal retinal laser remains an important primary treatment for DME. The ETDRS randomized 2244 patients with clinically significant macular edema (CSME) to either early treatment with focal and grid photocoagulation or deferral of treatment; and this landmark study showed at 3 years moderate visual loss was reduced by $50 \%$ with immediate focal argon photocoagulation treatment [1,3]. Visual acuity improved by more than 1 line in $40 \%$ of patients who underwent treatment compared with $22 \%$ in those who did not [1]. Diabetic macular edema occurs due to a malfunction of the blood-retinal barrier and death of endothelial cells leading to leakage of fluid and subsequent photoreceptor dysfunction [8]. Focal laser therapy leads to the occlusion of these leaking microaneurysms, pathologic vessels, or subretinal sites of leakage [6]. This closure is a delayed process that may extend up to 12 weeks after initial treatment [9]. As the ETDRS study showed, focal laser therapy can reduce moderate to severe vision loss, but the major effects were not seen till after 3 years of follow-up $[1,3]$.

The NAVILAS system has been shown to have both a high degree of safety and combines the advantages of computerized precision and accuracy to efficiently perform focal retinal laser therapy $[4-7,10,11]$. Traditional focal laser therapy requires a contact lens, topical anesthesia, and the patient's cooperation to fixate for the treatment period. Also, the ability of the retina specialist to locate each aneurysm based on the color fundus photograph or angiogram has the potential for treatment inaccuracy and often leads to physicians avoiding areas near the fovea, modifying their protocols and applying grid patterns [6], and performing several frequent retreatment sessions [12]. In comparison, retinal and eye-tracking systems such as the NAVILAS system align pre-planned treatment locations with the realtime fundus image increasing the potential effectiveness and efficiency [6,7]. Previously, it has been shown that a single focal therapy NAVILAS session can be efficiently completed including planning time in approximately 7 minutes and 47 seconds [4,7] and with significantly less pain compared to conventional laser treatment [10]. Similarly, all of our patients completed the NAVILAS treatment without complication and were also pleasantly surprised by the short duration of the procedure, the lack of discomfort, and the absence of a contact lens for the procedure.

Kozak et al. recently reported in June 2011 the accuracy of the NAVILAS system. In their analysis of the accuracy of 400 random focal targeted spots, they found that the system achieved a microaneurysm hit rate of $92 \%$ compared to the control group analyzing 100 focal spots performed manually, which was significantly lower at $72 \%$ [4]. Efficacy of NAVILAS focal laser treatment after 6 months showed statistically significant decrease in foveal thickness and improvement of visual acuity in 25 non-previously treated eyes with perifoveal telangiectasia $(n=3)$, central serious chorioretinopathy $(n=2)$, and diabetic macular edema $(n=20)$ [6]. 
Patients in our study reflect an average population at a public hospital with poorly controlled diabetes. Frequently, the disease is less than optimal controlled and treatment success of any retinal disease is limited by hyperglycemia, increased blood pressure, and renal compromise. In our current study, we showed the continued effectiveness of NAVILAS focal therapy after 12 months of follow-up. Even with poorly controlled diabetes with average hemoglobin A1C before and after treatment of 9.1 and 8.0, respectively, we observed a statistically significant decrease in macular edema and improvement of best-corrected vision after 12 months. In our cohort, 6 of the 7 patients had been treated previously with intravitreal bevacizumab, but only 3 patients required an average of 6.3 more injections after NAVILAS treatment, ranging from 21 to 263 days after treatment. Whether this decrease in number of patients receiving injections was related to the NAVILAS focal treatment remains unclear, but it is possible that a combination of focal therapy and intravitreal anti-VEGF can improve the treatment of DME. These initial findings from study infer that there is a potential improvement with combination focal NAVILAS and intravitreal anti-VEGF in the treatment of DME, but larger randomized controlled studies are necessary to confirm these findings.

Our results agree with another recently published study by Neubauer $e t$ al. [13]. They concluded that navigated laser therapy enables a reduced retreatment rate and faster visual acuity gain compared with conventional slit-lamp based laser. Although their cohort of 46 eyes including a larger sample size compared to our study, they only observed patients that had focal NAVILAS alone whereas our results incorporate patients that have been treated with anti-VEGF. As recommended by recent studies including the ranibizumab mono-therapy or combined with laser versus laser monotherapy for diabetic macular edema (RESTORE) study, anti-VEGF treatment combined with focal laser has become a prominent first line treatment for DME [2,14]. Our results suggest that using focal NAVILAS in conjunction with intravitreal anti-VEGF bevacizumab can potentially maintain visual outcomes for patients with diabetic macular edema. Future larger studies utilizing this novel, efficient focal laser delivery system with anti-VEGF treatment and the number of required post-NAVILAS focal laser intravitreal injections may be helpful in expanding our ability to efficiently treat DME.

There are several limitations to our current study including an overall small number of patients and the fact that patients were not randomized to treatment. Given that our study was a retrospective case series, we recommend further studies including a randomized treatment trial with focal NAVILAS and intravitreal anti-VEGF to draw definitive conclusions.

Focal retinal laser remains a long-term stabilization treatment for DME and is a proven therapy based on the initial ETDRS study. In this clinical series with 12 months of follow-up, we suggest that NAVILAS focal laser can be a safe and accurate alternative form of treatment for diabetic macular edema. Even with the development and increased usage of the intravitreal anti-VEGF therapy for macular edema, there still remains a role for focal. Combining these two modalities may increase the effectiveness of treatment and possibly lessen the long-term burden of continuous intravitreal anti-VEGF treatments.

\section{CONFLICT OF INTEREST}

No financial contributions to the work being reported or potential conflicts of interest.

Financial Disclosures include:

Dr. Jesse J. Jung - None.

Dr. Roberto Gallego-Pinazo - Novartis Pharmaceuticals Corporation (research funding, consultancy services, serving on speakers' bureaus, travel expenses); Alcon Laboratories, Inc. (travel expenses); Allergan (research funding); Bayer (research funding, consultancy services, travel expenses); Carl Zeiss Meditec (consultancy services, travel expenses)

Dr. Jonathan I. Huz - None.

Dr. Irene A. Barbazetto - None.

Dr. Antonio Lleó-Pérez - None.

\section{ACKNOWLEDGEMENTS} Inc.

This work was supported by The Macula Foundation,

\section{REFERENCES}

[1] ETDRS Research Group. Photocoagulation for diabetic macular edema: ETDRS Report 1. Arch Ophthalmol 1985; 103(12): 1796806.

[2] Nguyen QD, Brown DM, Marcus DM, et al. Ranibizumab for diabetic macular edema. Ophthalmology 2012; 119: 789-801.

[3] Blumenkranz MS. Optimal current and future treatments for diabetic macular oedema. Eye 2010; 24(3): 428-34

[4] Kozak I, Oster SF, Cortes MA, et al. Clinical evaluation and treatment accuracy in diabetic macular edema using navigated laser photocoagulator NAVILAS. Ophthalmology 2011; 118(6): 111924.

[5] Kernt M, Cheuteu R, Vounotrypidis E, et al. Focal and panretinal photocoagulation with a navigated laser $\left(\right.$ NAVILAS $\left.^{\circledR}\right)$. Acta Ophthalmol 2011; 89(8): e662-4.

[6] Kozak I, Kim JS, Oster SF, Chhablani J, Freeman WR. Focal navigated laser photocoagulation in retinovascular disease: clinical results in initial case series. Retina 2012; 32(5): 930-5.

[7] Chalam KV, Murthy RK, Brar V, Radhakrishnan R, Khetpal V, Grover S. Evaluation of a Novel, Non Contact, Automated Focal Laser with Integrated (NAVILAS) fluorescein angiography for diabetic macular edema. Middle East Afr J Ophthalmol 2012; 19(1): 158-62.

[8] Joussen A, Smyth N, Niessen C. Pathophysiology of diabetic macular edema. Dev Ophthalmol 2007; 39: 1-12.

[9] Gogi D, Gupta A, Gupta V, Pandav SS, Dogra MR. Retinal microaneurysmal closure following focal laser photocoagulation in diabetic macular edema. Ophthalmic Surg Lasers 2002; 33(5): 3627.

[10] Ober MD, Kernt M, Cortes MA, Kozak I. Time required for navigated macular laser photocoagulation treatment with the Navilas ${ }^{\circledR}$. Graefes Arch Clin Exp Ophthalmol 2013; 251(4): 104953.

[11] Kernt M, Cheuteu RE, Cserhati S, et al. Pain and accuracy of focal laser treatment for diabetic macular edema using a retinal navigated laser (Navilas). Clin Ophthalmol 2012; 6: 289-96. 
[12] Bamroongsuk P, Yi Q, Harper CA, McCarty D. Delivery of photocoagulation treatment for diabetic retinopathy at a large Australian ophthalmic hospital: comparisons with national clinical practice guidelines. Clin Exp Ophthalmol 2002; 30(2): 115-9.

[13] Neubauer AS, Langer J, Liegl R, et al. Navigated macular laser decreases retreatment rate for diabetic macular edema: a comparison with conventional macular laser. Clin Ophthalmol 2013; 7: 121-8.

[14] Mitchell P, Bandello R, Schmidt-Erfurth U, et al. The RESTORE study: ranibizumab monotherapy or combined with laser versus laser monotherapy for diabetic macular edema. Ophthalmology $2011 ; 118(4)$ : 615-25.

(C) Jung et al.; Licensee Bentham Open.

This is an open access article licensed under the terms of the Creative Commons Attribution Non-Commercial License (http://creativecommons.org/licenses/by$\mathrm{nc} / 3.0 /$ ) which permits unrestricted, non-commercial use, distribution and reproduction in any medium, provided the work is properly cited. 